\title{
Análise do perfil clínico e sociodemográfico dos pacientes pediátricos diagnosticados com glomerulonefrite difusa aguda em hospital no Sertão de Pernambuco, Brasil
}

Analysis of the clinical and sociodemographic profile of pediatric patients diagnosed with acute diffused glomerulonephritis in hospital in Brazil

Análisis del perfil clínico y sociodemográfico de pacientes pediátricos diagnosticados con glomerulonefritis aguda difusa en un hospital en Brasil

Rebeca Talita de Souza SIQUEIRA

Débora Rayssa Siqueira SILVA

Hellen Alves de CARVALHO

Pedro Lucas de Araújo ROCHA

Brena Paixão de Araújo SOUZA

Marília Soares SANTANA

Jéssika Cristina de LIMA

Pauliana Valéria Machado GALVÃo

Daniele Padilha LAPA

Patrícia de Moraes Soares SANTANA

Faculdade de Medicina (Campus Serra Talhada), Universidade de Pernambuco, UPE, 56909-535, Serra Talhada-PE, Brasil.

\section{Resumo}

Introdução: A glomerulonefrite difusa aguda é uma glomerulopatia decorrente do dano inflamatório dos componentes glomerulares, comumente encontrada em pacientes pediátricos, com padrão e prevalência variáveis de acordo com idade, sexo, fatores socioeconômicos e geográficos (principalmente nas glomerulonefrites infecciosas ou pós-infecciosas), sendo a causa mais comum de acometimento renal após os seis anos de idade. Porém, faltam dados mundiais precisos sobre a prevalência da síndrome. Objetivo: Traçar o perfil sociodemográfico e clínico dos pacientes pediátricos diagnosticados com esta glomerulonefrite no Hospital Regional Professor Agamenon Magalhães, localizado no município de Serra Talhada, Pernambuco. Material e método: Trata-se de um estudo retrospectivo e descritivo de corte transversal. A coleta de dados foi realizada entre agosto de 2018 a junho de 2019, através da análise dos prontuários dos pacientes pediátricos que foram diagnosticados com glomerulonefrite difusa aguda. Resultados: 29 prontuários foram analisados, correspondendo aos anos 2015 a 2018 , sendo 51,72\% do sexo masculino, residente em Serra Talhada e em zona rural, com idade média 7,7 anos, todos de etnia parda. Em admissão para atendimento hospitalar $75,86 \%$ da amostra apresentou estado geral regular, e apenas 3,45\% grave. 34,48\% da população estudada apresentou oligúria, 89,66\% edema generalizado, 58,62\% hipertensão arterial durante admissão, 27,59\% cefaleia e 55,17\% febre. Conclusão: Os resultados obtidos através da pesquisa destacam a importância de traçar o perfil para guiar os profissionais do serviço na assistência aos pacientes pediátricos acometidos pela patologia abordada.

Descritores: Glomerulonefrite; Glomérulos Renais; Pediatria.

\section{Abstract}

Introduction: An acute diffuse glomerulonephritis is a glomerulopathy caused by inflammatory damage to glomerular components, commonly found in pediatric patients, with a pattern and average prevalence according to age, sex, socioeconomic and geographic factors (mainly in infectious or post-glomerulonephritis). infectious), being the most common cause of renal impairment after six years of age. However, accurate global data on the prevalence of the syndrome are lacking. Objective: To draw the sociodemographic and clinical profile of pediatric patients diagnosed with this glome with this glomerulonephritis at the Regional Hospital Professor Agamenon Magalhães, located in city Serra Talhada, Pernambuco State, Brazil. Material and method: this is a retrospective and descriptive cross-sectional study. Data collection was carried out between August 2018 and June 2019, through the analysis of medical records of pediatric patients who were diagnosed by the acute diffuse glomerulonephritis. Results: 29 medical records were analyzed, corresponding to the years 2015 to 2018 , with $51.72 \%$ being male, living in Serra Talhada and in a rural area, with an average age of 7.7 years, all of mixed race. On admission to hospital care, $75.86 \%$ of the sample presented a general general condition and only $3.45 \%$ severe. $34.48 \%$ of the studied population had oliguria, $89.66 \%$ of generalized edema, $58.62 \%$ of arterial hypertension during admission, $27.59 \%$ of headache and $55.17 \%$ of fever. Conclusion: The results obtained through the research highlighted the importance of tracking the profile for professionals in the service of assistance to pediatric patients affected by the pathology addressed.

Descriptors: Glomerulonephritis; Kidney Glomerulus; Pediatrics.

\section{Resumen}

Introducción: Una glomerulonefritis aguda difusa es una glomerulopatía causada por daño inflamatorio a los componentes glomerulares, que se encuentra comúnmente en pacientes pediátricos, con un patrón y una prevalencia promedio de acuerdo con la edad, el sexo, los factores socioeconómicos y geográficos (principalmente en glomerulonefritis infecciosos o post-infeccioso), siendo la causa más común de insuficiencia renal después de los seis años de edad. Sin embargo, faltan datos globales precisos sobre la prevalencia del síndrome. Objetivo: Dibujar el perfil sociodemográfico y clínico de pacientes pediátricos diagnosticados con esta glomerulonefritis en el Hospital Regional Profesor Agamenon Magalhães, ubicado en el municipio de Serra Talhada, provincia de Pernambuco, Brazil. Material y método: se trata de un estudio transversal retrospectivo y descriptivo. La recolección de datos se llevó a cabo entre agosto de 2018 y junio de 2019 , a través del análisis de registros médicos de pacientes pediátricos que fueron diagnosticados por el glomerulonefritis aguda difusa. Resultados: se analizaron 29 registros médicos, correspondientes a los años 2015 a 2018, con un $51.72 \%$ de hombres, viviendo en Serra Talhada y en un área rural, con una edad promedio de 7.7 años, todos de raza mixta. Al ingreso a la atención hospitalaria, el $75.86 \%$ de la muestra presentaba un estado general general y solo el 3.45\% grave. El 34.48\% de la población estudiada tenía oliguria, $89.66 \%$ de edema generalizado, $58.62 \%$ de hipertensión arterial durante el ingreso, $27.59 \%$ de dolor de cabeza y $55.17 \%$ de fiebre. Conclusión: Los resultados obtenidos a través de la investigación resaltaron la importancia de seguir el perfil de los profesionales al servicio de la asistencia a pacientes pediátricos afectados por la patología abordada.

Descriptores: Glomerulonefritis; Glomérulos Renales; Pediatría.

\section{INTRODUÇÃO}

As glomerulopatias são um conjunto de síndromes clínicas, laboratoriais e histopatológicas primárias e secundárias a doenças sistêmicas que atingem os componentes filtrantes dos rins, os glomérulos. Estas podem ser causa frequente de doença renal crônica com necessidade de diálise (terapia de alto custo para o sistema de saúde), respondendo por $11 \%$ dos pacientes em tal 
tratamento no Brasil, e são as mais frequentemente diagnosticadas por biópsia renal, sendo, as formas primárias, responsáveis pelo acometimento de 2 a 3 indivíduos por 100.000 indivíduos no mundo ${ }^{1,2}$. Classificam-se como síndromes: nefríticas agudas ou glomerulonefrite difusa aguda; pulmonares-renais; nefróticas; da membrana basal; vasculares glomerulares; e associadas a doenças infecciosas ${ }^{3,4}$.

As glomerulonefrites decorrem de dano inflamatório que diminui a taxa de filtração renal, levando a retenção de componentes que deveriam ser excretados e possibilidade de insuficiência renal. $\mathrm{O}$ padrão histológico de acometimento é variável, podendo atingir os podócitos (células epiteliais das fendas de filtração), a membrana basal glomerular, o mesângio e endotélio. A maioria das glomerulonefrites é mediada por processos autoimunes, como depósitos e agressão por células do sistema imunitário, imunoglobulinas e complemento; outros participantes da patogênese incluem predisposição genética e infecções. As principais formas compreendem: glomerulonefrite difusa aguda pós-estreptocócica - GNPE (protótipo da GNDA pós-infecciosa); nefropatia por imunoglobulina A - IgA (doença de Berger); glomerulonefrite antimembrana basal glomerular (anti - MBG); glomerulonefrite ANCA-positiva; e nefrite lúpica ${ }^{4,5}$. Tais etiologias variam de acordo com as características dos pacientes. A síndrome nefrítica aguda clássica caracteriza-se por hematúria de início súbito oligúria, edema generalizado (muitas vezes periorbital), hipertensão arterial sistêmica acompanhada ou não de déficit leve da função renal. Apresenta-se, laboratorialmente, com: hematúria dismórfica, proteinúria subnefrótica (achado variável), componentes da via alternada do complemento diminuídos (C3 e CH50) e creatinina aumentada ou normal ${ }^{1,6}$.

A glomerulonefrite difusa aguda é comumente encontrada em pacientes pediátricos na prática de Pediatria e Nefrologia, com padrão e prevalência variáveis de acordo com idade, sexo, fatores socioeconômicos e geográficos (principalmente nas glomerulonefrites infecciosas ou pós-infecciosas), sendo a causa mais comum de acometimento renal após seis anos de idade. Porém, faltam dados mundiais precisos a respeito da prevalência da síndrome, como conjunto de diferentes etiologias. No Brasil, a GNDA constituiu o segundo diagnóstico mais frequente dentre as glomerulopatias primárias em Minas Gerais, entre os anos 1996 e 2010, e em crianças (11\%) em hospitais de referência de Salvador - BA, entre 2003 e $2016^{4,5,7,8}$.

A GNPE, protótipo das glomerulonefrites agudas pós-infecciosas, é uma complicação tardia não supurativa secundária ao contato ou infecção pelos estreptococos beta-hemolíticos do grupo A de
Lancefield (EBGA), caracterizada por inflamação de origem imunológica que afeta ambos os rins. É a glomerulopatia de maior ocorrência na infância, com incidência de 470 mil casos ao ano. Esta tem sido relatada raramente em países desenvolvidos. No entanto, em países subdesenvolvidos é uma síndrome epidêmica, com $97 \%$ dos casos ocorrendo nestes locais, o que corrobora para sua ligação com fatores socioeconômicos, sanitários e pela maior prevalência de piodermites causadas por estreptococos ${ }^{2,4}$.

A GNPE ocorre, com maior frequência, em crianças entre 2 e 14 anos de idade, com pico máximo aos 7 anos de idade; crianças do sexo masculino são as mais acometidas, numa proporção de 2:1, e não há predileção por etnia. Esse tipo de glomerulonefrite ocorre após períodos de incubação das cepas nefritogênicas de estreptococos betahemolíticos do grupo A que causaram infecções de pele e de garganta, sendo o impetigo a forma de infecção que mais comumente acometem as crianças residentes em regiões de altas temperaturas, como a região nordeste. A biópsia renal mostra proliferação de células do endotélio capilar e do mesângio com infiltrados leucocitários e depósitos de complexos imunes ${ }^{6}$.

Ademais, em um estudo realizado durante 18 anos (1998 - 2016) nos ambulatórios de referência em glomerulopatias de dois hospitais públicos de Recife, Pernambuco, foram achados 663 pacientes com essa enfermidade (36,8 casos/ano). Além disso, dentre os casos estudados, 112 (18\%) são de indivíduos com idade de até 19 anos, o que representa uma prevalência elevada de doenças glomerulares, especialmente as primárias (54-69\%), no estado pernambucano ${ }^{1}$.

Devido à frequência de acometimento, os levantamentos clínico e epidemiológico da GNDA em pacientes pediátricos permitem demonstrar sua frequência e as principais etiologias, contribuindo, assim, na definição de suas características clínicas, laboratoriais e histopatológicas no hospital regional da cidade de Serra Talhada, Pernambuco, bem como para auxiliar em estratégias de prevenção e terapia de tais entidades, contribuindo, assim, para evitar a evolução para glomerulonefrites crônicas, terceira causa de doença renal crônica em pacientes em diálise no Brasil ${ }^{1}$.

Dessa forma, ao se analisar o perfil sociodemográfico e clínico dos pacientes pediátricos diagnosticados com GNDA no Hospital Regional Professor Agamenon Magalhães (HOSPAM), localizado no município de Serra Talhada, Pernambuco, testa-se a hipótese de que, além de reafirmar a relação existente entre essas características epidemiológicas e a ocorrência de GNDA, ao serem feitos esses levantamentos, será possível criar um perfil dos pacientes pediátricos com risco de desenvolver essa doença na região. Assim, 
com tal perfil traçado, almeja-se melhorar a assistência e atenção à saúde a criança sertaneja.

\section{MATERIAL E MÉTODO}

Trata-se de um estudo retrospectivo, descritivo, observacional e transversal, cuja coleta de dados ocorreu nos meses de agosto de 2018 a junho de 2019. Foi realizada análise dos prontuários dos pacientes diagnosticados com Glomerulonefrite Difusa Aguda (GNDA), por meio das notificações realizadas nos anos de 2015 a 2018 na Enfermaria Pediátrica do HOSPAM, localizado em Serra Talhada, Pernambuco. A pesquisa respeitou as normas da Resolução $N^{o}$ 196/96 da Comissão Nacional de Ética em Pesquisa/Conselho Nacional de Saúde (CONEP/CNS), sendo aprovada por comitê de ética em pesquisa (CAAE: 91628618.9.0000.5207).

Foram incluídos no estudo crianças de ambos os sexos, com idade até 12 anos, diagnosticadas com GNDA no hospital supracitado. Foram excluídos do estudo os prontuários incompletos e as notificações duplicadas. As variáveis analisadas foram: 1. Sociodemográficas (idade, etnia, naturalidade e procedência); 2. Clínica (sinais e sintomas na admissão e durante o internamento, exames utilizados para diagnóstico, tratamento escolhido, complicações existentes e comorbidades associadas). A análise estatística dos dados ocorreu por meio do programa $R$ versão 3.6.1.

\section{RESULTADOS}

\section{- Características da população estudada}

Do total de 41 pacientes pediátricos notificados entre os anos de 2015 a 2018, 29 prontuários foram utilizados (os demais eram prontuários incompletos). Houve predomínio das seguintes características: pacientes do sexo masculino, residentes em Serra Talhada e provenientes da zona rural, na faixa etária de 2 a 12 anos de idade (média de 7,7 anos, mediana de 8 anos, desvio padrão 2,7 anos), todos de etnia parda. Percebeu-se que o maior número de casos ocorreu no ano de 2016. As demais características sociodemográficas da amostra estudada estão listadas na Tabela 1.

\section{- Dados clínicos da população estudada}

$\mathrm{Na}$ admissão para atendimento hospitalar, $75,86 \%$ (22) dos pacientes da amostra apresentaram estado geral regular, 20,69\% não obtiveram registro e apenas $3,45 \%$, estado geral grave. Dentre os sinais e sintomas apresentados relacionados à glomerulonefrite difusa aguda, foram encontrados, durante internamento hospitalar, em ordem decrescente de frequência: edema generalizado $(89,66 \%)$, hipertensão arterial na admissão $(58,62 \%)$, temperatura axilar maior ou igual a $37,7 \mathrm{C}(55,17 \%)$, oligúria $(34,48 \%)$ e cefaleia $(27,59 \%)$.

- Dados laboratoriais da população estudada $\mathrm{Na}$ avaliação laboratorial dos pacientes pediátricos registrada em prontuário, os exames presentes possuíam composição variável de acordo com a situação clínica e disponibilidade pelo serviço no período de internação dos pacientes. Dentre os exames solicitados que compõem métodos de auxílio para incrementar a investigação diagnóstica foram solicitados: sumário de urina, hemograma, anticorpo antiestreptolisina $\mathrm{O}$ (ASLO), proteína de fita urinária, ureia e creatinina (função renal). Os resultados encontrados estão apresentados na Tabela 2.

Tabela 1. Caracterização sociodemográfica da amostra estudada

\begin{tabular}{|c|c|c|}
\hline Variável & $\mathbf{n}$ & $\%$ \\
\hline \multicolumn{3}{|l|}{ Sexo } \\
\hline Masculino & 15 & 51,72 \\
\hline Feminino & 14 & 48,28 \\
\hline Total & 29 & 100,0 \\
\hline \multicolumn{3}{|l|}{ Ano de atendimento } \\
\hline 2015 & 6 & 20,69 \\
\hline 2016 & 10 & 34,38 \\
\hline 2017 & 8 & 27,59 \\
\hline 2018 & 5 & 17,24 \\
\hline Total & 29 & 100,0 \\
\hline \multicolumn{3}{|l|}{ Residência } \\
\hline Serra Talhada & 24 & 82,76 \\
\hline Triunfo & 2 & 6,90 \\
\hline Betânia & 1 & 3,45 \\
\hline Santa Cruz da Baixa Verde & 2 & 6,90 \\
\hline Total & 29 & 100,0 \\
\hline \multicolumn{3}{|l|}{ Zona } \\
\hline Zona Urbana & 14 & 48,28 \\
\hline Zona Rural & 15 & 51,72 \\
\hline Total & 29 & 100,0 \\
\hline
\end{tabular}

Tabela 2. Resultados de exames laboratoriais da população estudada

\begin{tabular}{|c|c|c|}
\hline Variável & $\mathbf{n}$ & $\%$ \\
\hline \multicolumn{3}{|l|}{ Hematúria } \\
\hline Sim & 18 & 62,07 \\
\hline Não & 8 & 27,59 \\
\hline Não informado & 3 & 10,34 \\
\hline Total & 29 & 100,0 \\
\hline \multicolumn{3}{|l|}{ Anemia } \\
\hline Sim & 20 & 68,97 \\
\hline Não & 9 & 31,03 \\
\hline Total & 29 & 100,0 \\
\hline \multicolumn{3}{|l|}{ ASLO reagente } \\
\hline Sim & 7 & 24,14 \\
\hline Não & 20 & 68,97 \\
\hline Não se aplica & 2 & 6,90 \\
\hline Total & 29 & 100,0 \\
\hline \multicolumn{3}{|l|}{ Proteinúria } \\
\hline Sim & 11 & 37,96 \\
\hline Não & 16 & 55,17 \\
\hline Não se aplica & 2 & 6,90 \\
\hline Total & 29 & 100,0 \\
\hline \multicolumn{3}{|c|}{ Insuficiência renal aguda (Creatinina alterada) } \\
\hline Sim & 7 & \\
\hline Não & 14 & \\
\hline Não se aplica & 8 & \\
\hline Total & 29 & 100,0 \\
\hline
\end{tabular}

\section{- Desfecho clínico}

A única complicação da síndrome nefrítica encontrada na amostra foi a insuficiência renal aguda, respondendo por $24,14 \%$ da população estudada. O tratamento individualizado realizado seguiu os protocolos da sociedade brasileira de pediatria, com uso de anti-hipertensivos e diuréticos de alça, administração de penicilina $\mathrm{G}$ benzatina, monitoração do peso e diurese durante internamento e rotinas pediátricas. Não houve encaminhamento para serviço especializado ou terciário de saúde e todos tiveram alta hospitalar com função renal preservada. 


\section{DISCUSSÃO}

As glomerulonefrites enquadram-se com $\mathrm{o}$ grupo de doenças representa a terceira principal causa de doença renal crônica no mundo, ficando atrás apenas da nefropatia diabética e das lesões renais associadas à hipertensão arterial. Adicionalmente, o Censo Geral dos Centros de Diálise, realizado pela Sociedade Brasileira de Nefrologia no ano de 2015, demonstrou que as glomerulonefrites constituíram $11 \%$ das doenças renais de base que levaram o paciente à necessidade de diálise ${ }^{9}$. No Brasil, a GNDA constituiu o segundo diagnóstico mais frequente dentre as glomerulopatias primárias em crianças nos hospitais de referência de Salvador BA, entre 2003 e $2016^{4,5,10}$.

Corroborando com o presente estudo, as glomerulonefrites de origem infecciosa são prevalentes em indivíduos do sexo masculino, com idade variando entre 6 e 10 anos; no entanto, pode incidir em qualquer faixa etária. Geralmente, os indivíduos adultos acometidos possuem alguma comorbidade prévia, como o diabetes mellitus. Quanto aos fatores socioeconômicos e geográficos, em países em desenvolvimento, a GNDA pós estreptocócica possui incidência anual que varia entre 9,5 a 28,5 casos por 100 mil habitantes, podendo corresponder a 50 a $90 \%$ dos casos de síndrome nefrítica na infância ${ }^{11}$.

As características encontradas na população do presente estudo estão em consonância com os dados mostrados na literatura. Em um estudo retrospectivo realizado em uma clínica pediátrica de nefrologia do Gaziantep University Hospital (Japão) com 75 pacientes diagnosticados com glomerulonefrite pós-infecciosa entre 2014 e 2016, $56 \%$ eram do sexo masculino e $44 \%$ do sexo feminino com idade média de $8,20 \pm 3,25 \operatorname{anos}^{12}$. Dados semelhantes foram encontrados em outro estudo, que analisou o prognóstico de crianças sudanesas acometidas por glomerulonefrite pósestreptocócica, o protótipo da glomerulonefrite difusa aguda pós-infecciosa; dessas crianças, $66,7 \%$ eram do sexo masculino ${ }^{13}$.

O quadro clínico é precedido por infecção estreptocócica (escarlatina, piodermite ou infecção de vias respiratórias superiores), respeitando um período de latência entre a ocorrência da infecção e a complicação tardia, que é a glomelunefrite pósinfecciosa, sendo caracterizado pela síndrome nefrítica aguda $^{10}$. Embora muitas doenças glomerulares tenham curso insidioso e assintomático, a síndrome nefrítica aguda caracteriza-se por hematúria de início súbito associada a oligúria, edema generalizado (muitas vezes periorbital) e hipertensão arterial sistêmica, acompanhada, ou não, de déficit leve da função renal. Quanto aos achados laboratoriais, a GNPE apresenta-se, em suma, com hematúria dismórfica, proteinúria subnefrótica (achado variável) e componentes da via alternada do complemento inicialmente diminuídos (C3 e CH50) ${ }^{1}$.

Os sinais e sintomas registrados no presente estudo são concordantes com o quadro clínico de pacientes de outras pesquisas. Em estudo com população semelhante, houve registro de edema $(86,7 \%)$ e hipertensão $(73,3 \%)$ como sinais mais comuns $^{12}$.

Os achados laboratoriais aqui encontrados também foram concordantes com os achados da literatura, corroborando com os dados encontrados ao diagnóstico da síndrome estudada. Hematúria, anemia e proteinúria de fita foram os achados mais comuns. De modo semelhante, em uma análise com pacientes com características semelhantes mostrou que, nos exames laboratoriais de crianças diagnosticadas com GNDA pós-infecciosa, a hematúria macroscópica foi o achado mais comum, acometendo $82,7 \%$ das mesmas; $77,3 \%$ tiveram proteinúria e $98,7 \%$ apresentaram complemento reduzido às custas de $\mathrm{C} 3^{12}$.

A GNPE é, caracteristicamente, uma doença que reduz os níveis de proteínas do complemento da via alternativa, principalmente o $\mathrm{C} 3$. Em dois terços dos casos, é possível identificar a presença de marcadores de infecção pregressa por estreptococos. No entanto, vale ressaltar que nem todos os estreptococos são nefritogênicos; o grupo A de Lancefield é frequentemente mais associado às lesões glomerulares ${ }^{10}$.

A presença de ASLO reagente pode indicar infecção estreptocócia (EBGA) prévia de orofaringe e faz parte de uma das provas para documentar a glomerulonefrite pós-infecciosa. No entanto, a glomerulonefrite decorrente de infecção prévia de pele (piodermites ou impetigo) por cepas nefritogênicas do EBGA é a mais comum na população de regiões com clima de altas temperaturas, como no Brasil, especialmente na região nordeste, justificando a baixa taxa de ASLO reagente no presente estudo, já que as infecções orofaríngeas são mais comuns durante o inverno e em regiões de clima frio ${ }^{2,4,6}$.

Em um estudo realizado de 2013 a 2014, para registro de características clínicas, investigações bioquímicas e sorológicas de crianças no sul da índia, região com temperaturas semelhantes às da região onde residiam os pacientes do presente estudo foi registrado que, das crianças com síndrome nefrítica aguda, $86,7 \%$ possuíam GNPE, principalmente com antecedente de piodermas e infecções respiratórias superiores, que precederam a hematúria em $80,6 \%$ e $5,6 \%$, respectivamente, dados que confirmam que as crianças com histórico de piodermite evoluem mais com GNPE ${ }^{14}$.

Sabe-se que a maioria dos pacientes com GNDA, principalmente GNPE, possuem bom prognóstico, mesmo aqueles que apresentam lesão 
renal aguda e crescentes na biópsia renal ${ }^{10}$. Porém, complicações sistêmicas graves podem desenvolverse durante o período de seguimento em pequena parcela dos indivíduos com GNPE. No presente estudo, das possíveis complicações da síndrome, a única presente foi a azotemia renal à custa da elevação da creatinina em pequena parte da população estudada, com evolução para recuperação e alta hospitalar sem necessidade de procedimentos invasivos. Dado semelhante foi encontrado em estudo realizado para avaliação do prognóstico de crianças acometidas por GNDA, no qual $29 \%$ apresentavam doença aguda grave; $87 \%$ das crianças estudadas recuperaram a função renal ${ }^{13}$. Esses dados mostram a progressão benigna da doença na maioria das crianças acometidas.

O manejo terapêutico registrado nos prontuários analisados se baseou no uso de penicilina $\mathrm{G}$ benzatina intramuscular com o objetivo de diminuir a disseminação de cepas nefritogênicas em caso de possibilidade de portar bactérias na pele ou na orofaringe. A antibioticoterapia profilática pode também ser indicada para os contactantes domiciliares ou em situações epidêmicas. Para atenuar a congestão cardiocirculatória e a hipertensão arterial, além da restrição hídrica e da dieta restrita em sódio, usou-se diuréticos de alça (furosemida), por via oral, na dose de $1-2 \mathrm{mg} / \mathrm{kg} / \mathrm{dia}$, como descrito na literatura ${ }^{15}$.

Algumas limitações encontradas neste estudo foram: ausência de teste diagnóstico para detectar queda dos níveis de complemento C3, exame que auxilia a firmar o diagnóstico da síndrome, dessa forma o diagnóstico dos pacientes foi dado de maneira sindrômica; ausência de prontuários digitais, que dificultou a busca de informações em prontuários manuais em papel, arquivados em caixas, sem setor próprio para o armazenamento; prontuários incompletos.

Pretende-se dar continuidade ao trabalho para conseguir amostragem maior e ampliar as variáveis estudadas para uma melhor comparação com os dados estatísticos da literatura, bem como relatar manejo da síndrome.

CONCLUSÃO

Ao se analisar o perfil sociodemográfico e clínico dos pacientes pediátricos diagnosticados com Glomerulonefrite Difusa Aguda (GNDA) no Hospital Regional Professor Agamenon Magalhães, localizado no município de Serra Talhada, Pernambuco, foi possível reafirmar a relação existente entre essas características epidemiológicas e a ocorrência de GNDA, ao serem feitos esses levantamentos, visto que as glomerulonefrites de origem infecciosa são prevalentes em indivíduos do sexo masculino, com idade variando entre 6 e 10 anos, no entanto, podendo incidir em qualquer faixa etária.
Com relação às complicações da síndrome nefrítica, este estudo evidenciou a insuficiência renal aguda como sendo a principal, sendo o tratamento individualizado, seguindo os protocolos da Sociedade Brasileira de Pediatria, com uso de anti-hipertensivos e diuréticos, monitoração do peso e diurese durante internamento e rotinas pediátricas, uma vez que não houve encaminhamento para serviço especializado ou terciário de saúde.

Devido à frequência de acometimento, os levantamentos clínico e epidemiológico da GNDA em pacientes pediátricos permitem demonstrar sua frequência e as principais etiologias, contribuindo, assim, na definição de suas características clínicas, laboratoriais e histopatológicas, bem como para auxiliar em estratégias de prevenção e terapia de tais entidades.

\section{REFERÊNCIAS}

1. Costa DMN, Valente LM, Gouveia PAC, Sarinho FW, Fernandes GV, Cavalcante MAGM, et al. Análise comparativa de glomerulopatias primária e secundária no nordeste do Brasil: dados do Registro Pernambucano de Glomerulopatias - REPEG. J. Bras. Nefrol. 2017; 39(1): 29-35.

2. Sim JJ, Batech M, Hever A, Harrison TN, Avelar T, Kanter MH, et al. Distribution of biopsy-proven presumed primary glomerulonephropathies in 20002011 among a racially and ethnically diverse US population. Am J Kidney Dis. 2016; 68(4): 533-544.

3. Crensiglova C, Rehme BB, Kinasz LRS, Chula DC, Do Nascimento MM, Soares MFS. Frequência e avaliação clínico-histológica das doenças glomerulares em um hospital terciário da região Sul do Brasil. J Bras Nefrol. 2016; 38(1): 42-48.

4. Kasper DL, Fauci AS, Hauser SL, Longo DL, Jameson JL, Loscalzo J. Manual de Medicina de Harisson. 19. ed. Porto Alegre: Mc Graw Hill/ Artmed; 2017.

5. Couser WG. Patogênese e tratamento da glomerulonefrite, uma atualização. J Bras Nefrol. 2016; 38(1): 107-122.

6. Figueira F, Alves JGB, Ferreira OS, Maggi RRS, Correia JB. Pediatria. 4a edição. Rio de Janeiro: MedBook; 2011.

7. Moorani KN, Sherali AR. Histopathological pattern in childhood glomerulonephritis. J Pak Med Assoc. 2010; 60(12): 1006.

8. Rocha LP, Carminati CR, Machado JR, Laterza VL, Reis MA, Corrêa RRM. Prevalence of nephropathies in children and adolescents and alterations in renal biopsies in Minas Gerais, Brazil, from 1996 to 2010. Ann Diagn Pathol. 2017; 17(1): 22-27.

9. Silva VS, Hagemann R, Viero RM. Glomerulonefrites primárias. In: Riella MC. Princípios de Nefrologia e Distúrbios Hidroeletrolíticos. Rio de Janeiro: Guanabara Koogan; 2018.

10. Bertola EA, Simonetti GD, Del Giorno R, Giannini $\mathrm{O}$, Fossali EF, Meoli M, et al. Extrarenal Immune- 
mediated disorders linked with acute poststreptococcal glomerulonephritis: a systematic review. Clinic Rev Allerg Immunol. 2019; 57(2): 294-302.

11. Sethi S, Fervenza FC. Standardized classification and reporting of glomerulonephritis. Nephrol Dial Transplant. 2018; 34(2): 193-199.

12. Kılıc BD, Akbalık MK, Buyukcelik M, Balat A. Pediatric post-streptococcal glomerulonephritis: Clinical and laboratory data. Pediatr Int. 2018; 60(7):645-650.

13. Ali el-TM, Babikir AM, El-Assad S, Abdelrahim MB. Prognosis of acute post-streptococcal glomerulonephritis in Sudanese children. Arab J Nephrol Transplant. 2014;7(2):103-107.

14. Gunasekaran K, Krishnamurthy S, Mahadevan S, Harish BN, Kumar AP. Clinical characteristics and outcome of post-infectious glomerulonephritis in children in Southern India: a prospective study. Indian J Pediatr. 2015; 82(10): 896-903.

15. Maia MLA, Vale MLD, Hatanaka E. Recomendações: Atualização de Condutas em Pediatria. Departamento de Nefrologia. Síndrome nefrítica. Departamentos Científicos SPSP, n. 88, p.10-14, 2019.

\section{CONFLITO DE INTERESSES}

Os autores declaram não haver conflitos de interesse.

\section{AUTOR PARA CORRESPONDÊNCIA}

\section{Pauliana Valéria Machado Galvão}

Rua Padre Bernardino Pessoa, 277, apto 801, Boa Viagem 51020-210 Recife - PE, Brasil

E-mail: pauliana.galvao@upe.br

Submetido em 26/05/2020

Aceito em 06/10/2020 